\title{
¿Hay alguna razón para cambiar la formadón médica en la Universidad española? Aportaciones de la Atención Primaria
}

\author{
A. de Lorenzo-Cáceres, E. Calvo Corbella* \\ M édico de Familia. Centro de Salud Universitario "Ciudad J ardín". Prof. A sociado. \\ Departamento de M edicina. Facultad de M edicina. Universidad Autónoma de \\ Madrid. *M édico de Familia. Centro de Salud Universitario "Pozuelo I". Madrid. \\ Miembro del Grupo de Trabajo de Pregrado de la Sociedad Española de M edicina \\ Familiar y Comunitaria (semFYC)
}

\section{RESUMEN}

La Medicina de Familia (MF) y la Atención Pri maria (AP) son fundamentales en el contexto de las reformas que necesita la formación médica en la Universidad española. Después de revisar la situa ción de la formación médica pregraduada en Espa ñ y los numerosos problemas que le aquejan, se repasan las grandes reformas educativas que la formación médica ha ido experimentando a lo lar. go del siglo XX, así como los diferentes modelos de currículo y las tendencias de las reformas que, en los últimos veinte años, han emprendido las Facul . tades de Medicina y los países de mayor prestigio, tanto en el ámbito académico universitario como en el de la ciencia médica y la organización sanita ria.

Se describe la denominada "enseñanza en la co munidad" (community-based teaching), así como sus peculiaridades y su grado de implantación en países como los EE.UU., Reino Unido o los Países Bajos. Se exponen algunas de las aportaciones que la AP y la MF hacen a la formación de los estu. diantes de Medicina y algunos de los retos y limita ciones que les plantea su plena incorporación a la docencia en el pregrado.

Por último, se analiza la situación de la MF en la Universidad española y se detallan alguna de las estrategias planteadas para conseguir la incor poración efectiva de la AP y la MF a nuestras Facultades de Medicina.

Palabras clave: Atención Primaria. Medicina de Familia. Educación médica. Pregrado. Enseñanza en la comunidad.
Is there any reason why to change medical edu cation at Spanish University? Primary Health Care contributions

\section{ABSTRACT}

Family Medicine and Primary Health Care are fundamental elements within the context of the re forms that are needed in medical education at Spa nish universities. After examinig the undergraduate medical education and the various problems afflic ting it, the great reforms that the studies of Medici ne have undergone throughout the twentieth cen tury are reviewed. Included are some examples of syllabuses and tendencies of the reforms that have been undertaken in the past 20 years be Medical Schools and countries of the highest prestige, not only at an academic level but in medical science and health organization as well.

The so-called Community-Based Teaching is described, along with its peculiarities and its de gree of establishment in countries like the U.S.A., the U.K. or the Netherlands. Some of the contribu tions that Primary Health Care and Family Medi cine have on the education of medical students and some of the challenges and limitations caused by their full incorporation into undergraduate tea ching are shown.

Finally, the situation of Family Medicine at Spa nish universities is analyzed, and some of the stra tegies put foward in order to achieve an effective incorporation of Primary Health Care and Family Medicine into our Medical Schools are detailed.

Key words: Primary Health Care. Family Medi cine. Medical education. Undergraduate medical education. Community-based teaching. 


\section{INTRODUCCIÓN}

Nuestra experiencia cotidiana en la docencia de estudiantes de $6^{\circ}$ curso de una de las Facultades de Medicina con más prestigio del país, la Universidad Autónoma de Madrid (UAM), nos sume año tras año en la misma perplejidad. Nuestros alumnos se sienten frustrados por una formación médica teórica y, cada vez más, enciclopédica, impartida a lo largo de un sobrecargado, agotador y selectivo plan de estudios, sin que nadie se sienta responsable de orientarles en habilidades básicas como las de entrevista clínica o la exploración física general de un paciente, mucho menos en el aprendizaje de técnicas elementales (medición de la tensión arterial, suturas y vendajes, aplicación de tratamientos por vía parenteral, etc.). Su experiencia clínica, basada en prácticas hospitalarias, les hacen concebir los pacientes como simples "portadores de casos", tanto más interesantes cuanto mayor reto y complejidad diagnóstica planteen, perdiendo poco a poco el componente de humanidad esencial con el que iniciaron ilusionados sus estudios. La transmisión de actitudes hacia el paciente, la profesión y el trabajo cotidiano, fundada en el papel de espejo de los docentes clínicos, les aleja de los principios de la ética médica basada, entre otros, en el respeto de la autonomía de los pacientes. De sus profesores, muy pocos han mostrado interés hacia ellos como personas, intentando aclararles dudas en momentos de desánimo, o ayudándoles a resolver problemas personales en años críticos de sus vidas. Con todo, a pesar de dejar en el camino a una porción de sus compañeros de estudios, la mayoría logra superar todas estas adversidades, obtiene una plaza de Médico Interno Residente (MIR) y se convierten en profesionales con una competencia clínica aceptable.

Algunos autores ${ }^{1-3}$ aseguran que nuestras Facultades de Medicina preparan opositores para la prueba de acceso a plazas MIR y que el MIR soluciona el fracaso de la educación médica del pregrado. Si al final todo se arregla, ¿hay alguna razón para cambiar nuestro modelo de formación médica pregraduada?

\section{LA FORMACIÓN MÉDICA EN EL PREGRADO: SITUACIÓN ACTUAL EN ESPAÑA}

Los problemas que presentan las Facultades de Medicina en España han sido identificados y repetidamente expuestos desde hace años por voces autorizadas, procedentes de los propios estamentos académicos ${ }^{1-5}$, quienes han llegado a decir que "la gran asignatura pendiente de la educación médica en España es el periodo de pregrado" añadiendo que, con el modelo educativo actual, no es posible conseguir el objetivo de formar médicos útiles a la sociedad $^{1}$.
El objetivo de este trabajo no es realizar un estudio detallado de las causas y consecuencias de los problemas que aquejan a nuestras Facultades de Medicina. Por ello, en la tabla $\mathrm{I}^{1-16}$ resumimos los problemas más relevantes y sobre los que existe un mayor consenso.

Sin embargo, todos los autores citados son conscientes de la dificultad de poner en práctica soluciones a los problemas detectados. La autonomía universitaria, las luchas de poder entre departamentos y su propia supervivencia, conforman una situación con pocos visos de introducir reformas en profundidad en nuestra formación médica de pregrado. Antes, se argumentaba que "había demasiados alumnos" para intentarlo. Ahora se alega la existencia del examen MIR como disculpa de una formación teórica basada en la adquisición de conocimientos ${ }^{1}$. Con todo, incluso tomada la decisión de una reforma en profundidad, serían necesarias unas condiciones mínimas previas para iniciar el camino del cambio ${ }^{3}$.

La posibilidad de que la solución provenga "desde fuera" de la Universidad también resulta remota. La presencia de representantes sociales en los órganos de dirección no pasa de ser testimonial y no parece que exista una demanda social para un cambio profundo en la formación de nuestros estudiantes de Medicina. Tras la descentralización autonómica, las respectivas administraciones educativas y sanitarias no han demostrado suficiente interés en el asunto. Es probable que, en algún momento, se plantee la reforma de la prueba MIR $^{17}$. Sin embargo, es difícil que en ella se plantee evaluar habilidades o actitudes, en las que el alumno no ha sido formado durante el pregrado. Por todo lo anteriormente expuesto, ¿hay razones para la esperanza?

Tal vez haya que esperar a la intervención de alguna directiva de la Unión Europea o al influjo de la docencia de la Medicina en países de nuestro entorno aunque, hasta la fecha, ninguna ha tenido repercusión práctica en los nuevos planes de estudio.

En el supuesto de que poco a poco se vayan produciendo las condiciones para el cambio, ¿hacia dónde debería orientarse? ¿Cuáles son las tendencias actuales en la formación médica? Antes de exponerlas, sería conveniente conocer cómo han evolucionado las ideas sobre la formación médica en el pregrado hasta llegar a la situación actual.

\section{EVOLUCIÓN DE LOS MODELOS DE ENSEÑANZA DE LA MEDICINA DURANTE EL SIGLO XX}

Desde que en 1910 el conocido Informe Flexner $^{18}$ recomendó reformas sustanciales en la formación médica en las medical school de los EE.UU., han sido varias las grandes reformas introducidas en los estudios universitarios de Medicina ${ }^{19}$. 
Tabla I

\section{PROBLEMAS DE LA FORMACIÓN MÉDICA PREGRADO EN ESPAÑA}

Problemas derivados de la definición de los objetivos docentes y de la propuesta curricular

Falta de definición y consenso institucional sobre los objetivos educativos (modelo profesional que la sociedad necesita), los conocimientos esenciales, las habilidades fundamentales que deben fomentarse, así como de la distribución de la carga lectiva entre las diversas disciplinas ${ }^{3}$.

Persistencia de currículos basados en disciplinas independientes ( 5 facultades $)^{2}$

Falta de verdadera integración de contenidos y objetivos docentes en los llamados "currículos integrados".

Fracaso de los "nuevos" planes de estudio (1990) (carga docente excesiva; rechazo de los departamentos a perder horas lectivas o contenidos; materias optativas no contempladas en la valoración de postgrado, ... $)^{2}$. "Peso excesivo de las asignaturas, los profesores, las cátedras y los aspectos cognitivos"

Modificación de los "nuevos" planes de estudio (1999) que no afronta los problemas planteados ${ }^{6}$.

Objetivos centrados en la adquisición de conocimientos ${ }^{1,3}$. Contenidos excesivos y, muchas veces, irrelevantes ${ }^{2}$.

Falta de objetivos basados en el aprendizaje de habilidades y actitudes.

Falta de acuerdo sobre los objetivos docentes y distribución de la carga docente entre preclínicos y clínicos ${ }^{5,7-9}$. Enseñanza y aprendizaje orientados a que la mayor proporción posible de alumnos de cada facultad solventen la prueba de acceso a la formación $M \mathrm{IR}^{1-3}$.

Falta de enseñanza orientada al autoaprendizaje como base de la formación continuada en el postgrado ("aprender a aprender") $)^{1-3}$

Discordancia en el concepto que alumnos ${ }^{10}$ y docentes ${ }^{8}$ tienen de la "excelencia" docente.

Problemas derivados de la metodología docente

Aprendizaje basado en la adquisición pasiva de conocimientos³.

Aprendizaje de conceptos utilizando, casi exclusivamente, clases magistrales.

Enseñanza centrada en el profesor y en el hospital ${ }^{3}$.

Presencia irrelevante de aprendizaje basado en resolución de problemas ${ }^{11,12}$ y en la toma de decisiones clínicas ${ }^{1}$ Durante las "prácticas" preclínicas, ausencia de formación en habilidades de utilidad en la práctica profesional ${ }^{2,5}$ Indefinición del tipo de habilidades que deben adquirirse durante las "prácticas" clínicas (rotaciones) y de los encargados de la formación en las mismas ${ }^{2 \cdot 3,13}$.

Mínima utilización de metodologías docentes avanzadas ${ }^{1}$. Uso inadecuado de los materiales audiovisuales ${ }^{14}$

Falta de preparación del profesorado en metodología docente ${ }^{3}$.

Problemas derivados de la organización de las estructuras universitarias y sanitarias

Doble dependencia del profesorado (Educación-CCAA / Sanidad-Servicios de Salud) ${ }^{6}$

Organización de ciclos independientes preclínico y clínic $0^{5}$

Areas de conocimiento, y departamentos, comunes a varias titulaciones biosanitarias: falta de orientación médica de los docentes de disciplinas básicas ${ }^{15}$. Proporción decreciente de médicos clínicos en los departamentos de ciencias básicas $^{9}$

Masificación de las facultades ${ }^{1}$

Falta de adecuación entre el número de admitidos con las necesidades futuras de profesionales ${ }^{1}$

Plazas de profesorado convocadas por necesidades docentes y seleccionadas, casi exclusivamente, por méritos de investigación ${ }^{2,9}$

Falta de reconocimiento de los méritos como docente para la promoción profesional ${ }^{4,6}$. Evaluación de los docentes, y financiación de los departamentos, en función de la actividad investigadora, sin relación con su calidad o excelencia como docentes

Falta total de coordinación entre las tres etapas de la formación médica (pregrado, postgrado y continuada) ${ }^{3}$

A diferencia del papel promotor de la reforma en otros países (EE.UU., Gran Bretaña, Holanda), nula influencia de la Organización Médica Colegial por su tradicional falta de representatividad y de innovación profesional

Mínimo impacto de la representación social en los órganos de gobierno universitario, sin que se fomente la reorientación del "producto" de la docencia universitaria, intentando adelantarse y dar respuesta a los continuos cambios sociosanitarios y, así, buscar el mayor beneficio de la colectividad 
Tabla I

PROBLEMAS DE LA FORMACIÓN MÉDICA PREGRADO EN ESPAÑA (CONTINUACIÓN)

Problemas derivados de los sistemas de evaluación

Evaluación exclusiva de conocimientos, fundamentalmente basado en aprendizaje memorista de detalles poco relevantes, y evaluados con preguntas de opción múltiple

Ausencia de evaluación sistemática y estructurada de habilidades clínicas (ECOE) $)^{16}$ y de actitudes profesionales

Falta de evaluación de la calidad como docentes del profesorado

Falta de evaluación y control social de la consecución de los objetivos inicialmente planteados por los planes de estudios

En un artículo anterior ${ }^{20}$ se comentaron las principales reformas implantadas en los currículos* de formación médica pregraduada. En la tabla II $^{18,21-28}$ se resumen las fechas y reformas más relevantes. Básicamente, los principales modelos curriculares son los siguientes ${ }^{5,29}$ :

1. Currículos paralelos o clásicos, basados en disciplinas independientes. Todavía cinco facultades españolas continúan con estos currículos ${ }^{2}$.

2. Currículos secuenciales o integrados: contenidos organizados por aparatos o sistemas: Western Reserve University (EE.UU.), década de los 50.

-Integración horizontal: coordinación de las asignaturas de un mismo curso

- Integración vertical: coordinación de asignaturas situadas en cursos diferentes.

3. Currículos basados en la resolución de problemas ${ }^{11,30-33}$ : McMaster University (Canadá), 1968; Harvard (EE.UU.); Maastrich (Países Bajos).

4. Nuevos currículos:

-Currículo integrado basado en presentaciones clínicas $^{34,35}$ : Calgary (Canadá), 1995.

- Currículos orientados a las funciones profesionales de los médicos ${ }^{21,36-38}$ : proyecto EFPO de las cinco universidades del estado de Ontario (Canadá), 1990-92.

Remitimos al lector interesado a la lectura de la bibliografía seleccionada para conocer sus características, ventajas y limitaciones.

Conocida la evolución que ha ido sufriendo la concepción del currículo en la formación médica de pregrado, ¿cuáles son, actualmente, las grandes tendencias educativas?

\section{TENDENCIAS ACTUALES EN LA FORMACIÓN MÉDICA DE PREGRADO}

Desde 1986, en ámbitos preocupados por las estrategias educativas en ciencias de la salud ${ }^{39}$, la filosofía que representa la nueva orientación de la enseñanza de postgrado es conocida por el acrónimo inglés SPICES, ya que las características principales que la definen son:

\footnotetext{
*Currículo: Plan de Estudios (D.R.A.E., 20a ed. Madrid, 1992).
}

-(S) Centrada en el estudiante (student) y no en el profesor.

-(P) Basada en la resolución de problemas, no en la transmisión de información.

-(I) Integrada, no basada en asignaturas o disciplinas. tales.

-(C) Orientada a la comunidad, no a los hospi-

-(E) Basada en cursos optativos (electivity), no en currículos estándar.

-(S) Sistemática y no oportunista.

- Además debe ser multiprofesional, no uniprofesional.

García Barbero et al. ${ }^{40}$ describen con detalle todas estas estrategias, sus ventajas e inconvenientes.

En los últimos veinte años, se han producido una serie de innovaciones y reformas docentes que podríamos agrupar en, al menos, cinco grandes tendencias. Estas tendencias hacen referencia a:

1. Definición de objetivos docentes en función de lo que la sociedad necesita del médico, permitiendo además la adaptación de dichos objetivos a los cambios sociales acelerados.

2. Modificación de la metodología empleada en la docencia de la Medicina.

3. Importancia de la adquisición de habilidades, no sólo clínicas, sino de comunicación y útiles para el posterior mantenimiento de la competencia profesional ("aprender a aprender").

4. Importancia de la adquisición de actitudes, definidas como "aprendizaje del oficio"1 o "profesionalismo" $"$.

5. Importancia de disponer de nuevas herramientas de evaluación. Hasta el momento, se han desarrollado fundamentalmente para la evaluación de habilidades clínicas (OSCE: Objetive Structured Clinical Examination $)^{16,42}$ y de comunicación ${ }^{43-45}$.

Algunas de las innovaciones o realizaciones en cada uno de estos cinco apartados se presentan en la tabla III ${ }^{1,13,16,21,30-32,36,41,43-45,46-60}$.

Ahora bien, dentro de este mundo en transformación, ¿cuál es el lugar de la Medicina de Familia y de la Atención Primaria en las nuevas tendencias de la formación de pregrado? 
Tabla II

HITOS EN LA FORMACIÓN MÉDICA EN PREGRADO EN EL SIGLO XX

INTERNACIONAL

1910. Informe Flexner ${ }^{18}$

Unión de ciencias básicas y clínicas. Prácticas con pacientes en hospitales clínicos universitarios.

1932. Comisión de Formación Médica de la asociación Americana de Colegios Médicos (AACM $)^{21}$

Necesidad de reformar el énfasis en contenidos memoristas y no en recursos intelectuales de significado permanente, desarrollo de capacidad de autoaprendizaje útil durante toda la vida profesional.

1969. Todd Report 22

En medicina general se aprenden habilidades que no se aprenden en el hospital, por lo que se recomienda la presencia del estudiante en las consultas de MG.

Informes 1983 y 1988 de la Marcy Foundation para la reforma en EE.UU.

1984. World Health Organization, Office for Europe. Primary Health Care in undergraduate medical education Copenhage, $1984^{23}$

1988. Reunión de ministros europeos de Educación y Sanidad. Lisboa.

1988 Declaración de Edimburgo ${ }^{24}$

Prioridad: utilización de todos los recursos sanitarios de la comunidad, no sólo los hospitales, para la formación médica de pregrado.

1990 Proyecto ACME-TRI (Assessing Change in Medical Education - The Road to Implementation) de la A.A.M.C.

(Association of American Medical Colleges) ${ }^{25}$

Necesidad de cambio en la educación médica

1991 OMS. Changing Medical Education.

1993 Tomorrow's Doctors (General Medical Council) ${ }^{26}$

Recomendaciones para la educación médica de pregrado: la enseñanza debe adaptarse a los cambios en la atención de los pacientes.

1993 Directiva 16/ 93 (Consejo CEE), sobre estudios de pregrado en Medicina.

1994 Conferencia OMS / WONCA (Ontario) ${ }^{27}$

Recomendaciones $11,12,13,14$ y 17 .

1996 Directiva XV/ E/ 8443/ 2/ 95-ES. Comité consultivo Formación Médicos. Comisión Europea (Bruselas)²8.

NACIONAL: El caso de la U.A.M.

1983. Ley 11/ 83 de Reforma Universitaria (LRU). Boletín Oficial del Estado (BOE) 209, 1 sept. 1983.

1986. Ley 14/ 86 General de Sanidad (LGS). BOE 102, 29 abril 1986.

1986. Real Decreto (RD) 1558/ 86. Conciertos Universidades e Instituciones Sanitarias. BOE 182, 31 jul. 1986.

1987. RD 1497/ 87. Directrices generales comunes de los planes de estudio. BOE, 14 dic. 1987.

1987. Requisitos de los Centros de Atención Primaria asociados a la Universidad. Orden del 31-7-87. BOE 188/87.

1988. Primera rotación obligatoria por AP en la UAM.

1990. RD 1417/90. Título universitario oficial de Licenciado en Medicina y directrices generales de los planes de estudio conducentes a su obtención. BOE 278, 20 nov. 1990.

1992. 10 C. de S. universitarios y 10 profesores asociados en 1992.

1993. Nuevo Plan de estudios Fac. Medicina UAM. BOE 227/93. 22 sept. 1993.

1994. Orden 8309. Convenio INSALUD UAM. BOE 88, 13 abril 1994.

1999. Reforma del "nuevo" plan de estudios de la UAM. Resolución 1-9-99. BOE 222/ 99. 


\section{Tabla III}

\section{LAS CINCO GRANDES TENDENCIAS EN LAS REFORMAS DE LOS CURRÍCULOS DE LA FORMACIÓN MÉDICA EN EL PREGRADO}

Objetivos dirigidos a las necesidades de la sociedad

Definición de competencias profesionales ${ }^{21,36}$.

Empleo sistemático de nuevas metodologías docentes

Aprendizaje Basado en Problemas ${ }^{30-32}$

Uso de pacientes y familias estandarizados ${ }^{46-47}$

Método clínico centrado en el paciente ${ }^{48}$

Aprendizaje Basado en la presentación de casos (Case-Based) ${ }^{49}$.

Modelos simulados para aprendizaje de habilidades clínicas ${ }^{50}$ :

Simulador de patología cardiovascular multimedia.

Reanimación Cardio-Pulmonar (R.C.P.)

Algunas técnicas quirúrgicas: cirugía menor, infiltración de partes blandas

Uso de nuevas tecnologías ${ }^{51}$ :

Enseñanza asistida por ordenador (Computer-assisted learning): uso de PC y sistemas multimedia (CD-ROM) $)^{52}$

CACC (Computer Assisted Clinical Case)

Recursos on-line en Internet: Virtual Patient Projet ${ }^{53}$

Videograbación:

Vídeos utilizados para la docencia

Uso de videograbación en enseñanza de habilidades de comunicación (Rol Play o entrevistas reales)

Metodología para la enseñanza-aprendizaje para la adqusición de actitudes ${ }^{54}$

Énfasis en la adquisición de habilidades

Habilidades clínicas ${ }^{13,55}$.

Habilidades de comunicación y entrevista clínica ${ }^{43}$.

Aprender a aprender: Formación Médica Continuada (FMC).

Medicina Basada en Pruebas (MBE) y Lectura crítica de artículos ${ }^{56}$ :

Cómo formular preguntas relevantes

Cómo buscar la mejor información

Cómo analizar críticamente la información seleccionada

Cómo utilizar la información buscada

Diseño de protocolos de investigación

Habilidades de presentación oral y de redacción científica

Uso de inglés

Importancia en la adquisición de actitudes

"Profesionalismo" 41.

Subordinación de los propios intereses (abnegación)

Subordinación a estrictos principios morales y éticos

Respuesta a las necesidades sociales

Acreditar, en la práctica, un espíritu de valores humanistas

"Aprendizaje del oficio" ${ }^{1:}$

Motivación

Iniciativa

Responsabilidad

Pensamiento crítico

Conciencia ética

Dedicación: puntualidad y asistencia

Relaciones con pacientes y familiares

Nuevos sistemas de evaluación

Evaluación de actitudes ${ }^{57}$

Métodos estandarizados de evaluación:

De habilidades clínicas: OSCE (Objetive Structured Clinical Evaluation) ${ }^{16}$.

De habilidades de comunicación y entrevista clínica ${ }^{43-45}$.

Otras

Introducción de disciplinas no tradicionales:

Ética Médica ${ }^{58}$

Consejo y Asesoría (Counsselling) ( $^{5}$

Diversidad Cultural - Multiculturalismo ${ }^{60}$

MBE (Medicina Basada en Pruebas)

Genómica, etc. 


\section{LA AP / MF EN LA FORMACIÓN MÉDICA DE PREGRADO}

En los nuevos currículos, se da una importancia creciente a la Medicina de Familia y a la Atención Primaria, debido a la implicación que tiene en las cuatro primeras tendencias anteriormente reseñadas. Por ello, queremos detenernos especialmente en una de las innovaciones más significativas de las emprendidas en las dos últimas décadas, la denominada Community-Based Teaching (C-B.T.) o Enseñanza-Aprendizaje en la Comunidad.

\section{Community-Based Teaching (C-B. T.)}

Desde finales de los años ochenta, diversas instancias educativas y sanitarias ${ }^{24-26,61-63}$ han apremiado a los responsables de la formación médica de pregrado para utilizar todos los recursos educativos disponibles. En particular, se ha hecho hincapié en la necesidad de que los estudiantes realicen buena parte de su formación en estructuras asistenciales comunitarias o ambulatorias ${ }^{64,65}$. Por su importancia cuantitativa y cualitativa trataremos, fundamentalmente, de la docencia en Centros de Atención Primaria y con médicos generales / de familia. Para ello, exponemos de forma breve la situación en algunos de los países con mayor implantación de la C-B.T:

-EE.UU.: desde 1980 se viene realizando la formación de estudiantes por los diferentes médicos "generalistas" de EE.UU. ${ }^{66,67}$. Se calcula que el $30 \%$ de los médicos de familia y el $20 \%$ de los internistas "generalistas" (con práctica ambulatoria) desarrollan labores como docentes "voluntarios" (no forman parte de los departamentos universitarios) colaborando en la docencia de los estudiantes ${ }^{68}$.

Al menos el 75\% de las 125 Facultades de Medicina norteamericanas realizaban estancias obligatorias de 6-8 semanas con médicos de familia ${ }^{69}$. Dadas las peculiaridades de la práctica asistencial estadounidense (práctica privada), incluso se ha analizado el impacto económico que le representa al médico la presencia de estudiantes en la consulta (aumento del tiempo total de consulta, reducción en el número de pacientes citados, coste atribuible a la docencia $\left.{ }^{70-72}\right)$. El American College of Physi cians (AMC) no es ajeno a esta tendencia. Tanto el $\mathrm{AMC}^{73}$ como otros autores ${ }^{74}$, han elaborado interesantes documentos para orientar a los médicos generales con práctica asistencial en los objetivos educativos, requisitos como docentes y métodos de evaluación en C-B.T.

Dentro del amplio abanico de los actuales planes de estudio de las facultades norteamericanas ${ }^{75}$ se han desarrollado diversas experiencias de integración de la Atención Primaria en los currículos, tanto longitudinales ${ }^{76}$ como transversales ${ }^{77}$.

-Gran Bretaña: siguiendo las recomendaciones del Colegio Oficial de Médicos ${ }^{26}$ los departamentos universitarios de Atención Primaria (Ge neral Practice / Primary Health Care / Familiy Medicine) han desarrollado una gran diversidad de actividades formativas en la comunidad ${ }^{78,79} \mathrm{de}$ manera que, probablemente, sea en el Reino Unido donde la enseñanza en la comunidad (C-B.T.) ha alcanzado su mayor difusión y rigor metodológico. En 1998, todas las facultades contaban con departamentos de AP/MG, participando de manera significativa en la carga curricular (en 1999, hasta el 9,6\% de las horas docentes en el Imperial College, London. Berlin A. Comunicación no publicada).

La propia "explosión" de la docencia en AP por médicos de familia/generales (GP) no está exenta de problemas estructurales y riesgos ${ }^{80,81}$, como son la existencia de un gran número de tutores colaboradores que forman un departamento "virtual" repartido en un gran número de centros asistenciales, la dificultad de mantener la competencia y motivación docente de los profesores a largo plazo o las dificultades para financiar y proporcionar tiempo protegido para la docencia entre otras.

-Países Bajos: la universidad holandesa ha seguido la tradición del país de situarse a la vanguardia en un amplio espectro de diferentes movimientos y decisiones sociales. La Universidad de Maastrich, por ejemplo, fue una de las primeras en el mundo en definir un currículo basado en la resolución de problemas. Por otra parte, el Colegio de Médicos Generales holandeses goza de un reconocido prestigio a la hora de establecer estándares de calidad asistencial. Tras la publicación del Blueprint holandés ${ }^{82}$, en el que se definían los objetivos de la educación médica en el pregrado para las universidades holandesas, Martens et al ${ }^{83}$ (Universidad de Maastrich) identificaron los requisitos estructurales, objetivos educativos, habilidades y actitudes susceptibles de aprendizaje durante las rotaciones por Atención Primaria con médicos generales.

En la tabla $\mathrm{IV}^{12,84-98}$ se resume la situación actual de los Departamentos Universitarios de Medicina de Familia/Medicina General/ Atención Primaria tanto en el extranjero como en España.

A la vista de estos datos, podríamos preguntarnos cuál es el motivo del vertiginoso desarrollo de la formación médica de pregrado en la comunidad en los últimos años, de forma que cada vez hay más universidades que complementan en Centros de Atención Primaria una buena parte de la formación clínica hasta entonces asumida por los hospitales universitarios. 
Tabla IV

\section{LA AP EN LA FORMACIÓN MÉDICA DE PREGRADO}

ESPAÑA

En 1999, en España existían ${ }^{84}$ :

Alrededor de 150 médicos de familia que son profesores asociados en, al menos, 17 Universidades (p.e. 17 en U.A.M., 15 en Sevilla, 12 en la Universidad Central de Barcelona (U.C.B.)).

Más de 100 Centros de Salud Universitarios (p.e 18 en la UAM, 15 Sevilla, 10 Alicante).

Rotaciones clínicas 85.89 en Centros de Salud en 24 Facultades de Medicina. La mayoría son obligatorias, y tienen una duración entre 32 h y 2-3 meses. Habitualmente se sitúan en 6 curso.

Existe una asignatura obligatoria (no troncal) de MF/ AP en las Universidades de Sevilla9 ${ }^{90}$ Castilla-La Mancha (Albacete) y Barcelona (U.C.B.) ${ }^{91}$.

En varias Universidades existen asignaturas optativas con contenidos propios de MF/AP: UAM ${ }^{12,}$ Alicante ${ }^{92}$, U.C.B., Valladolid, Lérida.

Las Facultades de Medicina de las Universidades Autónomas de Madrid (UAM) y de Barcelona (UAB) publicaron la reforma del "nuevo plan de estudios" en 1999, a los 6 años del inicio del plan anterior.

8 Facultades tienen pendiente de desarrollar sus "nuevos" planes de estudios desde 1993.

\section{OTROS PAÍSES}

\section{EUROPA}

17 países con departamentos universitarios de Medicina General / de Familia.

No existen departamentos en España, Grecia e Italia.

GRAN BRETAÑA $93-96$

1962: primer catedrático de un Departamento de Primary Health Care - General Practice.

1998: última de las 24 facultades británicas en nombrarlo.

Estructura de un Departamento de General Practice (Imperial College. London, 1999):

2 Catedráticos

2 Titulares "senior" +8 Titulares

120 General Practitioner tutores (60 urbanos +60 rurales)

Existen 121 tutores de método clínico y 22 de "contacto inicial" con el paciente

Promoción 1997-2002: 15\% horas currículo en/ por AP

Financiación/ Presupuesto: 1 millón de libras/año

\section{EE.UU. ${ }^{97}$}

En 1996, 112 de 125 Facultades con Departamento de MF.

$75 \%$ tiene asignaturas clínicas y rotaciones obligatorias por MF (6-8 semanas) en 3ㅇ curso ( $1^{\text {er }}$ año de clínica) ${ }^{98}$.

La mayoría ofrecen contenidos en los dos primeros años (preclínicos). Más del $90 \%$ con cursos optativos en el cuarto año.

\section{¿Qué aporta la AP en la formación de pregrado?}

En la introducción de este artículo, ya hemos expuesto las opiniones acreditadas que expresaban la necesidad de cambio desde dentro de la propia Universidad española. Los cambios sociales del último cuarto de siglo han hecho necesarios numerosos cambios en las organizaciones de asistencia sanitaria: estancias hospitalarias cada vez más cortas con cuidados ambulatorios cada vez más frecuentes; ingreso de pacientes cada vez más graves y más seleccionados; progresivo envejecimiento de la población, aumentando la prevalencia de enfermedades crónicas; crecimiento de la necesidad de cuidados a domicilio, incluidos los cuidados palia- tivos; necesidad de desarrollar una mejor comunicación médico-paciente e importancia del abordaje psicosocial para la atención adecuada de todos los procesos; necesidad de cuidados multiprofesionales y en equipo; protagonismo del paciente en la valoración y control de su propio proceso ${ }^{99}$. Todos estos aspectos han justificado la necesidad de trasladar buena parte de la formación de los estudiantes de Medicina desde los hospitales hacia la Atención Primaria

En este contexto, la Medicina de Familia se sitúa en un lugar privilegiado para aportar soluciones a los numerosos problemas que se le plantean a la formación médica de pregrado. La experiencia de otros países durante los últimos veinte años nos 
permite deshacer algunos de los "mitos" que se relacionan con la educación médica de pregrado en la AP. Así:

- La enseñanza en la comunidad no es más cara que en los hospitales ${ }^{100}$.

-En AP se pueden aprender, no sólo habilidades de comunicación, prevención o promoción de la salud ${ }^{80}$, sino habilidades clínicas, tanto de anamnesis como de exploración clínica ${ }^{101,102}$. Muchas de las habilidades son adquiridas con igual éxito tanto en el hospital como en AP. Otras son más fácilmente adquiridas en alguno de los dos ámbitos ${ }^{103}$.

-En aquellos países donde, como en los EE.UU., existe un interés institucional ${ }^{68}$ en fomentar la elección de especialidades "generalistas" entre los estudiantes que acceden a los programas de especialización de postgrado, la AP en el pregrado se ha revelado como un medio idóneo de lograr ese objetivo institucional. En EE.UU. existen, al menos, cuatro iniciativas de ámbito nacional (Genera list Curriculum Project de la Health Resources and Services Administration; Generalist Physician Initiative Robert Wood Johson Foundation ${ }^{104}$, Pew Charitable Trusts y WK Kellogs Foundation) que proporcionan recursos para desarrollar currículos universitarios basados en C-B.T.

—La AP no sólo es útil para alumnos que deben o desean ser médicos de familia. También ha demostrado su utilidad para contribuir en la formación de otros especialistas médicos (pediatras, cirujanos, psiquiatras, etc.), así como en la de otras profesiones sanitarias, como odontólogos o enfermeras.

\section{¿Qué tipo de recursos docentes utiliza la AP en el pregrado?}

El tipo de colaboración docente con la Universidad más extendido en la AP española es la estancia o rotación de estudiantes junto a médicos de familia en Centros de Salud (CS) ${ }^{105}$. Sin embargo, existen otras formas de participación docente que vienen siendo utilizadas por los departamentos universitarios y profesores de AP de otros países (Berlin A. Comunicación no publicada). Así, podemos mencionar:

-Enseñanza "tradicional" de una disciplina académica de AP / MF (asignaturas, seminarios, talleres,..)

- Conocimiento individual de los pacientes en su medio familiar, domicilio, medio social, etc. son frecuentes las iniciativas para promover un "contacto precoz" entre el estudiante y los "pacientes" (no hospitalizados) desde los primeros cursos de licenciatura (preclínicos) ${ }^{106}$.

-Enseñanza "compartida" con otras disciplinas. La AP puede aportar pacientes no hospitalizados y con hallazgos clínicos estables para la enseñanza de habilidades clínicas de materias especializadas, como auscultación de soplos en valvulopatías reumáticas, exploración de pacientes hipertensos, diabéticos, etc.

-Enseñanza por MF de temas comunes a varias disciplinas: técnicas de comunicación, ética médica, consejo, modificación de hábitos, etc.

Volviendo a nuestro país, debemos reconocer que el ejemplo de lo ocurrido en países tomados como modelo en otros aspectos de la organización sanitaria y de la atención médica, no parece haber tenido ningún efecto sobre la formación pregraduada en el ámbito de la Atención Primaria, ni sobre la presencia de la Medicina de Familia en nuestros currículos. ¿Cuál es la situación actual? ¿Qué estrategias se han propuesto para introducir la Medicina de Familia en nuestras facultades? ¿Cuál es nuestra propuesta?

\section{PAPEL DE LA AP EN LA REFORMA DE LA FORMACIÓN MÉDICA DE PREGRADO EN ESPAÑA}

Aunque la situación actual ${ }^{84-92,107}$ era impensable hace quince años, debemos convenir que tiene pocos visos de cambiar de forma significativa, habiendo llegado a un cierto punto de equilibrio donde, lo más que es posible lograr en la práctica, es la introducción de alguna "asignatura obligatoria" o alguna plaza más de profesor asociado. Llegados a este punto, ¿debiéramos aspirar a algo más que a departamentos o cátedras de Medicina de Familia?

En los últimos años, las voces más representativas del ámbito de la Medicina de Familia, y que están más estrechamente vinculadas con el mundo universitario, han expuesto su opinión sobre el camino a seguir.

\section{Estrategias propuestas para la consolidación de la MF en la Universidad española}

La Sociedad Española de Medicina Familiar y Comunitaria, tanto a través de diversas iniciativas (Grupo de Trabajo de Docencia en Pregrado, Mesa de Pregrado en las Jornadas Docentes de los Congresos Nacionales de la SemFYC, Jornadas Universidad y Medicina de Familia ${ }^{108}$ ), como a través de diferentes comunicados institucionales ${ }^{109} \mathrm{y}$ documentos $^{110}$, ha pretendido hacer llegar su voz hasta los ámbitos de responsabilidad sobre la formación de pregrado en España. Sin embargo debemos reconocer que, aunque sus relaciones institucionales con las diversas administraciones sanitarias han resultado eficaces en algunas ocasiones, su nivel de influencia en el entorno académico es mínimo. 
Se ha propuesto ${ }^{111}$ que la estrategia básica para incorporar de forma eficaz la Medicina de Familia en la Universidad sería lograr, como primer paso, que ésta se incluyese en el catálogo de áreas de conocimiento del Consejo de Universidades, ya que éste es el paso normativo para poder contar, a medio plazo, con departamentos de Medicina de Familia en nuestras facultades. Estos autores han elaborado una abundante documentación (científica, histórica, jurídica y administrativa, tanto nacional como internacional) con la que fundamentar la consideración de la Medicina de Familia como un área de conocimiento independiente.

Otros expertos en la materia ${ }^{12,113}$, abogan por definir una estrategia común a nivel estatal, abordando el proceso de integración de la MF en la Universidad desde perspectivas operativas, no conceptuales o teóricas. Opinan, además, que debiera mejorarse la información que sobre la MF y los médicos de familia tienen los demás profesionales de la Medicina (incluidos los docentes universitarios). Del mismo modo, es preciso mejorar las deficiencias formativas de los médicos de familia en su conjunto, aumentando su competencia profesional y el impacto de su actividad investigadora.

Algunos autores ${ }^{99}$ consideran que, en la situación actual, el objetivo estratégico es conseguir la aparición de plazas de profesores numerarios con el perfil explícito de Atención Primaria y no tanto conseguir el reconocimiento de un área de conocimiento específica. Dichas plazas deberían adscribirse a alguna de las áreas de conocimiento ya existentes. Para la enseñanza de pregrado sería más trascendente que la Medicina de Familia aportara su capacidad para apoyar los aspectos generales de la formación médica en lugar de impartir los aspectos específicos de su especialidad. De igual modo, sería prioritario aunar esfuerzos para conseguir plazas de profesores numerarios, en lugar de aspirar a lograr la aparición de asignaturas obligatorias de Atención Primaria o Medicina de Familia. Llegan a proponer, incluso, que la denominación específica de las plazas y del área de conocimiento sea la de Atención Primaria en lugar de Medicina de Familia. Además, opinan que la docencia en pregrado debería convertirse en una opción específica en la carrera profesional de médicos de familia especialmente motivados y capacitados.

\section{Nuestra propuesta}

Con anterioridad a este artículo, se ha sugerido ${ }^{99}$ que "la Medicina de Familia verá facilitada su entrada en la Universidad, como una estructura per- manente, en la medida en que se identifique con las nuevas tendencias en la educación médica de pregrado y, así, poder demostrar sus grandes posibilidades de colaborar en el logro de los nuevos objetivos docentes que se plantean en esta época de cambio".

Tales objetivos podrían ser: la adquisición de habilidades de comunicación, el protagonismo del paciente y de los factores psicológicos y socioculturales del mismo, el énfasis en el enfoque comunitario, en la promoción de la salud y en la prevención de la enfermedad, aprender a trabajar en equipo, etc.

En esta línea, pensamos que la Medicina de Familia y la Atención Primaria debieran plantearse su incorporación a la Universidad española en un contexto ligado a la reforma en profundidad de los actuales currículos. Si nuestras aspiraciones y reclamaciones se limitan a lograr la existencia de asignaturas, departamentos, profesores o catedráticos, sin cuestionar el planteamiento actual de la docencia, seremos una más en la larga lista de disciplinas aspirantes a su reconocimiento académico (Geriatría, Alergología, Oncología, Bioética, etc.) que reclaman con argumentos similares su consideración como áreas de conocimiento.

Una opción que no debiéramos olvidar es la posibilidad de establecer contactos con universidades de otros países de nuestro entorno donde sí tiene protagonismo la Medicina de Familia, bien como área de conocimiento o como departamento universitario. Así, podría darse la paradoja de que participáramos tan sólo en la clásica rotación de alumnos en los Centros de Salud asociados a nuestras facultades y estuviéramos unidos mediante proyectos de investigación -realizando así actividades propias de la carrera profesional universitaria- en una comunidad virtual con facultades lejanas en el espacio, pero más próximas a nuestras aspiraciones que la Universidad española. Por ejemplo, la estancia durante periodos breves en universidades europeas para realizar estudios, fellowship en investigación, tesis doctorales o aprender metodología y organización docente es una realidad que podría satisfacer actualmente las aspiraciones académicas de los médicos de familia.

\section{Retos y problemas del futuro}

Muchos son los retos que nos plantea asumir este planteamiento. Probablemente sea más sencillo conseguir alguna plaza como profesores universitarios que crear una infraestructura de Centros de Salud y de docentes preparados en las nuevas metodologías docentes para alcanzar, como han demostrado en la práctica en otros países ${ }^{101-102}$, que el aprendizaje en AP de todos los estudiantes de 
nuestras facultades sea igual o superior que en los actuales hospitales universitarios.

Los problemas que pueden presentarse en el futuro no deben ser muy diferentes de los detectados ya en EE.UU. ${ }^{114}$ o Gran Bretaña ${ }^{80,81}$ y que ya hemos esbozado con anterioridad. La escasa implantación actual de la MF en el pregrado en España puede representar un factor añadido para la aparición de nuevos problemas. Así, podemos señalar:

-El escaso impacto, en la práctica, de la docencia de pregrado dentro del colectivo de los médicos de familia (150 profesores asociados frente a 2.000 tutores de postgrado, entre los 15.000 especialistas actuales). Buena prueba de ello lo constituye la escasa repercusión interna que se deriva de actividades anuales como las Jornadas Universidad y Medicina de Familia o las Mesas de Pregrado de los Congresos Nacionales.

-Falta de preparación en nuevas tendencias y metodologías docentes en pregrado (no necesariamente coincidentes con las habilidades docentes útiles en la formación de postgrado).

- Las redes asistenciales no contemplan un "tiempo protegido" para la docencia (tampoco existe en los hospitales). Dentro de las jornadas laborales cada vez más sobrecargadas, el estudiante resta tiempo de la asistencia, frente al residente que puede asumir parte de la misma.

- Son aún escasas las iniciativas de innovación docente. Hasta el momento, abundan las rotaciones por los CS o las propuestas de asignaturas frente a otras iniciativas educativas.

—Falta de profesionalización de la labor docente de pregrado. Ante la inexistencia de algún tipo de incentivo para los médicos asistenciales que son necesarios para participar en la docencia como profesores colaboradores, en algunos centros se utiliza la rotación periódica de la plaza de profesor asociado, con la discontinuidad que esta práctica representa. Además, la retribución que la Universidad concede a los profesores asociados puede considerarse como "testimonial", desligada de una adecuada evaluación de la competencia y dedicación docente, sin que suponga un estímulo para aquellos profesionales que pretenden dedicarse de un modo profesional a la docencia.

\section{CONCLUSIONES}

Pese a las reformas emprendidas por la Universidad española desde la promulgación de la Ley de Autonomía Universitaria en 1983, a pesar de las sucesivas modificaciones de los planes de estudio y de la opinión mayoritaria de que la formación universitaria de la Medicina en España está necesitada de profundas reformas, nos encontramos en un mo- mento en el que no parece haber motivos para confiar en un cambio significativo en la situación de estancamiento en la que se encuentran nuestras Facultades de Medicina.

La influencia de las directivas comunitarias y de la docencia de pregrado en los países de nuestro entorno, aquéllos que sirven de referencia tanto al mundo universitario como al sanitario, podrían constituir elementos dinamizadores de unas estructuras caracterizadas, clásicamente, por su resistencia al cambio y su alejamiento de las necesidades sociales.

En este contexto de necesidad de reformas, tanto de los currículos como de la concepción del proceso de enseñanza-aprendizaje y de las metodologías docentes empleadas, la Atención Primaria y la Medicina de Familia se encuentran en una posición privilegiada para aportar muchas de las soluciones y recursos que son necesarios para alcanzar los objetivos en la formación de los médicos que nuestra sociedad necesita ahora y los que necesitará en un inmediato futuro.

Dado el retraso en la incorporación de la Medicina de Familia a la formación de pregrado en España, consideramos que la participación de la Atención Primaria en la docencia universitaria debiera ir estrechamente ligada a planteamientos renovadores de la formación médica. Así, mantendríamos la línea seguida por nuestra especialidad en su proceso de implantación y desarrollo en nuestro país.

Partiendo con cierto retraso respecto a los países de nuestro entorno, la historia de la Medicina de Familia en España está indisolublemente unida a la Reforma Sanitaria de la Atención Primaria, siendo la primera especialidad en diseñar y unificar el programa docente para la formación de postgrado de sus residentes y en evaluar la competencia de los mismos al finalizar su formación. También lidera los programas de formación de tutores y la formación reglada mediante cursos complementarios durante el postgrado en materias olvidadas por nuestras facultades (Comunicación médico-paciente; Gestión sanitaria; Bioética; Metodología de la investigación, etc.).

Los fundamentos conceptuales y científicos en los que se basa la aspiración de incorporar la Medicina de Familia a la docencia universitaria están suficientemente acreditados. Sin embargo, es posible que un planteamiento restringido que circunscriba el debate a la necesidad de una disciplina universitaria de Medicina de Familia pueda malograr el compromiso de nuestra especialidad con el objetivo final de lograr una actividad universitaria que contribuya a la formación de los médicos que espera nuestra sociedad.

A los médicos de familia comprometidos en la docencia universitaria se nos plantea un reto apa- 
sionante. ¿Seremos capaces de hacer compatibles nuestras legítimas aspiraciones para ocupar el lugar que nos corresponde en la estructura docente de nuestras facultades de Medicina y, al mismo tiempo, promover y desarrollar las reformas en el proceso de enseñanza-aprendizaje que nuestros alumnos necesitan?

\section{CORRESPONDENCIA:}

Antonio de Lorenzo-Cáceres Ascanio

C.S. Universitario "Ciudad Jardín"

C/ Pantoja, 3

28002 Madrid

e-mail: med011366@nacom.es

\section{Bibliografía}

1. Rozman C. La educación médica en el umbral del siglo XXI. Med Clin (Barc) 1997; 108: 582-6.

2. Palés JL. Análisis del proceso de implantación de los nuevos planes de estudio en la Licenciatura de Medicina, en "Nuevas estrategias en la formación del médico del siglo XXI". $1^{a}$ ponencia del XIV Congreso Nacional de la Sociedad Española de Educación Médica. Educación Médica 1999; 2 (3): 102-4.

3. Oriol-Bosch A, Gual-Sala A. Consideraciones sobre enseñar a aprender medicina. JANO 1994; 47 ( $n^{\circ}$ extra. Dic): 10 S$16 \mathrm{~S}$.

4. Prat J. Bases para la elaboración de un nuevo plan de estudios en la Facultad de Medicina de la Universitat de Lleida (FM-UdL), en "Nuevas estrategias en la formación del médico del siglo XXI". $1^{a}$ ponencia del XIV Congreso Nacional de la Sociedad Española de Educación Médica. Educación Médica 1999; 2 (3): 107-8.

5. Iríbar MC, Campos AJ, Peinado JM. Integración preclínicaclínica en la enseñanza teórica y práctica de la medicina. Educación Médica 1999; 2 (4): 174-8.

6. Rozman C. Universidad de Ciencias de la Salud: ¿una opción innovadora?. Med Clin (Barc) 2000; 115: 381-3.

7. Rodés J, Trilla A. Fórmulas para la integración de la formación básica y clínica en medicina. Med Clin (Barc) 1999; 379-82.

8. Rodríguez Artalejo F, Benegas JR, Rodríguez Artalejo A, Rodríguez Artalejo C. Un modelo para el análisis de la actividad académica en medicina. Med Clin (Barc) 1997; 108: 499-502.

9. Ricoy JR, Carrasco M, Clavería LE. Educación médica e investigación. Med Clin (Barc) 1999; 259-63.

10. Lou JM, Zapatero MD, Alda JO, Giménez I, Escanero JF. El profesor de medicina del siglo XXI: expectativas del alumnado (resultados preliminares). Comunicación al XIV Congreso Nacional de la Sociedad Española de Educación Médica. Educación Médica 1999; 2: 149-50.

11. Baños JE. El aprendizaje basado en problemas (problembased learning): su empleo como método docente. Educación Médica 1999; 2 (4): 194

12. Lorenzo-Cáceres A de, Otero A, Calvo E, Engel JL. Contacto precoz con el paciente en atención primaria. Una experiencia de aprendizaje basada en problemas. Educación Médica 1998; 1: 24-31.

13. Peinado JM, Iribar MC, Metz J, Patricio M, Szekeres P. El proyecto Euroskills: a la búsqueda de un consenso europeo en la enseñanza de las destrezas clínicas. Comunicación al XIV Congreso Nacional de la Sociedad Española de Educación Médica. Educación Médica 1999; 2: 123.

14. Manso JM. Enseñanza de la Medicina y nuevas tecnologías ¿una huida hacia adelante?, en: "Aplicación de las nuevas tecnologías en la enseñanza de las ciencias de la salud. $2^{\text {a }}$ Ponencia del XIV Congreso Nacional de la Sociedad Espa- ñola de Educación Médica. Educación Médica 1999; 2 (3): 109-111.

15. García Olivares E. Enseñanza de asignaturas comunes a distintas licenciaturas. ¿Una enseñanza diferencial en Medicina?. Comunicación al XIV Congreso Nacional de la Sociedad Española de Educación Médica. Educación Médica 1999; 2 (3): 122-3.

16. Prat J, Mothe P, Galiano F, Viñas J, Martínez JM. Adquisición de competencias en pre y postgrado, valorada mediante evaluación clínica objetiva estructurada (ECOE - OSCE). Comunicación al XIV Congreso Nacional de la Sociedad Española de Educación Médica. Educación Médica 1999; 2: 145.

17. Fornells JM. Análisis crítico del sistema MIR, en "Análisis y perspectivas de la formación postgraduada". $3^{\mathrm{a}}$ Ponencia del XIV Congreso Nacional de la Sociedad Española de Educación Médica. Educación Médica 1999; 2 (3): 113-4.

18. Flexner A. Medical education in the United States and Canada: a report to the Carnegie Foundation for the advancement of teaching. New York, NY: Arno Press and The New York Times, 1972.

19. Christakis NA. The similarity and frequency of proposals to reform US medical education. Constant concerns. JAMA 1995; 274: 706-11.

20. Calvo Corbella E, Ferrándiz Santos J, Puche López N. ¿Es necesario incluir la Medicina de Familia / Atención Primaria en la licenciatura médica? MEDIFAM 1992; 2 (3): 156-61.

21. Neufeld VR, Maudsley RF, Pickering RJ, Turnbull JM, Weston WW, Brown MG, et al. La formación de los futuros médicos en Ontario. Educación Médica 2000; 3: 77-95.

22. Royal Comission on Medical Education. Todd Report (CMND 3569). Londres: HMSO, 1968.

23. World Health Organization. Regional Office for Europe. Primary Health Care in undergraduate medical education. Copenhague: WHO, 1984.

24. Metcalfe DH. Edinburgh declaration. Fam Pract 1989; 6: 165-7.

25. Mann KV, Kaufman DM. A response to the ACME-TRI report: the Dalhousie problem-based learning curriculum. Med Educ 1995; 29: 13-21.

26. General Medical Council. Tomorrow's Doctors: Recommendations on undergraduate medical education. Londres: General Medical Council, 1993.

27. OMS-WONCA. Haciendo el ejercicio médico y la formación médica más adecuados a las necesidades de la población: La contribución del médico de familia. Conferencia conjunta OMS-WONCA, Ontario (Canadá) 1994. Barcelona: SemFYC, 1996.

28. Comisión Europea: Comité consultivo para la formación de médicos. Directiva XV / E/ 8443/2/ 95-ES. Bruselas, 1996.

29. Nuevos horizontes en la enseñanza integrada de la medicina [editorial]. Educación Médica 1999; 2 (4): 157-8. 
30. Clarke R. Aprendizaje basado en la resolución de problemas. En: Cox KR, Evan CE, editores. La docencia en medicina. Barcelona: Doyma 1990; 55-62.

31. Neufeld VR, Woodward CA, McLeod SM. The McMaster MD programme: a case study in renewal in medical education. Acad Med 1989; 64: 423-32.

32. Albanese MA, Mitchell S. Problem-based learning: a review of literature on its outcomes and implementation issues. Acad Med 1993; 68: 52-81.

33. Roma i Millán J. La enseñanza basada en problemas y la enseñanza en grupo: una alternativa para estudiantes, residentes y profesionales, en "Nuevas estrategias en la formación del médico del siglo XXI". $1^{\text {a }}$ ponencia del XIV Congreso Nacional de la Sociedad Española de Educación Médica. Educación Médica 1999; 2 (3): 104-6.

34. Mandin H, Harasym P, Eagle Ch, Watanabe M. Developing a "clinical presentation" curriculum at the University of Calgary. Acad Med 1995; 70: 186-93.

35. Mandin H. Curricula for the education of medical students: conceptual guidelines for medical schools, en "Nuevas estrategias en la formación del médico del siglo XXI". $1^{\mathrm{a}}$ ponencia del XIV Congreso Nacional de la Sociedad Española de Educación Médica. Educación Médica 1999; 2: 106.

36. OMS. Introducción a la preparación de planes de estudio basados en la competencia. Cuadernos de Salud Pública 1978; 68: 23.

37. Manso Martínez JM. ¿Qué enseñar en ciencias de la salud?. Técnicas para definir competencias y perfiles profesionales ( $1^{\mathrm{a}}$ parte). Educación Médica 2000; 3: 61-8.

38. Manso Martínez JM. ¿Qué enseñar en ciencias de la salud?. Técnicas para definir competencias y perfiles profesionales (y II). Educación Médica 2000; 3: 101-6.

39. Harden RH, Sowdwn S, Dunn W. Some educational strategies in curriculum development: The SPICES model. Med Educ 1984; 18: 284-97.

40. García Barbero M, Alfonso Roca MT, Cancillo Salas J, Castejón Ortega JV. Planificación educativa en ciencias de la salud. Barcelona: Masson, 1994; 87-103.

41. Swick HM, Szenas Ph, Danoff D, Whitcomb ME. Teaching professionalism in undergraduate medical education. JAMA 1999; 282: 830-2.

42. Miller GE. The assessment of clinical skill competence performance. Acad Med 1990; 65 (9 Suppl.): 63S- 67S.

43. Hulsman RL, Ros WJ, Winnubst JA, Bensing JM. Teaching clinically experienced physicians communication skills. A review of evaluation studies. Med Educ 1999; 33: 655-68.

44. Cleries X, Kronfly E. El aprendizaje de habilidades de comunicación por parte de profesionales sanitarios: reflexiones a partir de diversas experiencias. Med Educ 2000; 3: 9-18.

45. Prados Castillejo JA, Ruiz Moral R, Borrell i Carrió F, Bosch Fontcuberta JM, Bellón Saameño JA, Perula de Torres L. Creación de un instrumento de medida de la comunicación médico-paciente. Validación para residentes. Comunicación al XIV Congreso Nacional de la Sociedad Española de Educación Médica. Educación Médica 1999; 2: 132-3.

46. Colliver JA, Barrows HS, Vu NV, Verhuslt SJ, Mast TA, Travis TA. Test security in examinations that use standarised-patient cases at one medical school. Acad Med 1991; 66: 279-82.

47. Pugnaire MP, Leong SL, Quirk ME, Mazor K, Gray JM. The standardized family: an innovation in primary care education at the University of Massachusetts. Acad Med 1999; 74 (1 Suppl.): 90S- 97S

48. Thistlethwaite JE, Jordan JJ. Patient-centred consultations: a comparison of student experience and understanding in two clinical environments. Med Educ 1999; 33: 678 -85.

49. Irby DM. Three exemplary models of case-based teaching. Acad Med 1994; 69: 947-53.
50. Issenberg SB, McGaghie WC, Hart IR, Mayer JW, Felner JM, Petrusa MR, et al. Simulation technology for health care professional skills training and assessment JAMA 1999; 282: 861-6.

51. Moberg TF, Whitcomb ME. Educational technology to facilitate medical students' learning: background paper of the medical school objectives project. Acad Med 1999; 74:1146-50.

52. Reid WA, Harvey J, Watson GR, Luqmani R, Harkin PJ, Arends MJ. Medical student appraisal of interactive computer-assisted learning programs embedded in a general pathology course. J Pathol 2000; 191: 462-5.

53. McGee JB. The Virtual Patient Projet. Using computer simulations for medical student education, en "Aplicación de las nuevas tecnologías en la enseñanza de las ciencias de la salud". $2^{\text {a }}$ Ponencia del XIV Congreso Nacional de la Sociedad Española de Educación Médica. Educación Médica 1999; 2: 111 .

54. Hensel WA, Rasco TL. Storytelling as a method for teaching values and attitudes. Acad Med 1992; 67: 500-4

55. Du Boulay C, Medway C. The clinical skills resource: a review of current practice. Med Educ 1999; 33: 185-91.

56. Taylor R, Reeves B, Ewings P, Binns S, Keast J, Mears R. A systematic review of the effectiveness of critical appraisal skills training for clinicians. Med Educ 2000; 34: 120-5.

57. Smith RC, Marshall AA, Cohen-Cole SA. The efficacy of intensive biopsychosocial teaching programs for residents: a review of the literature and guidelines for teaching. J Gen Intern Med 1994; 9: 390-6.

58. Fox E, Arnold RM, Brody B. Medical ethics education: past, present, and future. Acad Med 1995; 70: 761-9.

59. Moorhead R, Winefield H. Teaching counselling skills to fourth-year medical students: a dilemma concerning goals. Fam Pract 1991; 8: 343-6.

60. Loudon RF, Anderson PL, Gill PS, Greenfield SM. Educating medical students for work in culturally diverse societies. JAMA 1999; 282: 875-80.

61. World Federation for Medical Education. Procceedings of the World Summit on Medical Education. Med Educ 1994; 28 (Suppl 1).

62. Cohen JJ. Generalism in medical education: The next steps. Acad Med 1995; 70: 57-60.

63. Inui TS, Williams WT, Goode L, Anderson RJ, Bhak KN, Forsyth JD, et al. Sustaining the development of primary care in academic medicine. Working Group on Sustaining the Development of Academic Primary Care. Association of American Medical Colleges. Acad Med 1998; 73: 245-57.

64. Perkoff GT. Teaching clinical medicine in the ambulatory seetting: an idea whose time may have finally come. N Engl J Med 1986; 314: 27-31.

65. Matorras R. El entorno de la docencia [editorial]. Med Clin (Barc) 1989; 93: 175-7.

66. Yonke AM, Foley RP. Overview of recent literature on undergraduate ambulatory care education and a framework for future planning. Acad Med 1991; 66: 750-5.

67. Irby DM. Teaching and learning in ambulatory care setings: A thematic review of the literature. Acad Med 1995; 70 : 898-931.

68. Vinson DC, Padem C, Devera-Sales A, Marshall B, Waters EC. Teaching medical students in community-based practices: A national survey of generalist physicians. J Fam Pract 1997; 45: 487-94.

69. Khan NB. Medical Schools can indeed produce family physicians. Familiy Medicine, 1996; 28 (6): 439-40.

70. Vinson DC, Paden C, Devera-Sales A. Impact of medical student teaching on family physicians' use of time. J Fam Pract 1996; 42: 243-9.

71. Crouse BJ, Norris ThE, Wolff LTh. Rural physicians as educators: Why take on another job?. Am Fam Phy 1996; 54: $1457-60$ 
72. Fields SA, Toffler WL, Bledsoe NM. Impact of the presence of a third-year medical student on gross charges and patient volumes in 22 rural community practices. Acad Med 1994; 69: 587-9.

73. Deutsch SL, Noble J, editores. Community Based Teaching. A guide to developing and implementing education programs for medical students and residents in the practitioner's office. American College of Physicians, 1997. http://www.acponline.org/catalog/books/cbtguide.htm

74. Sheets KJ, Harris D1. Questions asked by familiy physicians who want to serve as medical student preceptors. J Fam Pract 1996; 42: 503-11.

75. Barzansky B, Jonas HS, Etzel SI. Educational programs in US medical schools, 1999-2000. JAMA 2000; 284: 1114-20.

76. Freeman J, Cash C, Yonke A, Roe B, Foley R. A longitudinal primary care program in a urban public medical school: Three years of experience. Acad Med 1995; 70 (1 Suppl.): 64S- 68S.

77. Bridge PD, Schenk M, Popp S. Evaluating a primary care vertically integrated curriculum in undergraduate medical education. Fam Med 2000; 32: 525-7.

78. Robinson LA, Spencer JA, Jones RH. Contribution of academic departments of general practice to undergraduate teaching, and their plans for curriculum development. Br J Gen Pract 1994; 44: 489-91.

79. Lefford F, McCrorie P, Perrin F. A survey of medical undergraduate community-bassed teaching taking undergraduate teaching into the community. Med Educ 1994; 28: 312-5.

80. Wilson A, Fraser R, McFinle RK, Preston-Whyte E, Whynn A. Undergraduate teaching in the community: Can general practice deliver?. Br J Gen Pract 1996; 46: 457-60.

81. Murray E, Modell M. Community-based teaching: The challenges. Br J Gen Pract 1999; 49: 395-8.

82. Metz JCM, Pels Rijcken EH, Brandvan den Valkenburg BWM. Training of Doctors. Blueprint 1994. Objectives of Undergraduate Medical Education in the Netherlands. Nijmegen: University Press, 1994.

83. Martens FMJG, van der Vleuten CPM, Grol RPTM, Root JMH op 't, Crebolder HFJM, Rethans J-J. Educational objectives and requirements of an undergraduate clership in general practice. The outcome of a consensus procedure. Family Practice 1997; 14 (2): 153-9.

84. Bonal Pitz P, Gil Guillén V, Martín Zurro A, Pinto Espanhol A, Calvo Corbella E. La Medicina de Familia como área de conocimiento. Aten Primaria 1999; 23: 151-74.

85. Otero A, Carreira J, Villamor J. Nuevas tendencias en la enseñanza de la medicina. Evaluación de la rotación en centros de salud. Aten Primaria 1992; 9: 512-5.

86. Menárguez JF, Saturno PJ, Gómez-Calcerrada D, Sánchez JA, Saura J. Evaluación de la calidad de las prácticas de atención primaria realizadas en centros de salud de Murcia. Rev San Hig Pub 1994; 68: 279-87.

87. Buitrago F, Vergeles JM, Cano-Hernández E. Evaluación de la rotación en centros de salud de los alumnos de la Facultad de Medicina de la Universidad de Extremadura. Aten Primaria 1994; 13: 118-24.

88. Orozco D, Gil VF, Quirce F. La experiencia en la Universidad de Alicante en la formación de pregrado en medicina familiar y comunitaria. Cuadernos de Gestión 1996; 2: 140-9.

89. Córdoba R, Melguizo A, Montón C, Concepción A de. Experiencia de la rotación por los centros de salud de los alumnos de sexto curso de la Facultad de Medicina de Zaragoza. Aten Primaria 1997; 19: 273.

90. Bonal P. Medicina familiar y comunitaria. Una asignatura universitaria. Aten Primaria 1994; 13: 392-6.

91. Casado Vicente V, Bonal Pitz P. Asignaturas de Medicina de Familia. Tribuna Docente 2000; 1: 3-8.

92. Gil VF, Quirce FF, Simón-Talero M, Orozco D, Uris J, Merino J. Medicina en la atención primaria (asignatura optativa de pregrado en la Universidad de Alicante): dos años de experiencia. Aten Primaria 1993; 12: 152-4.
93. Hannay DR, Campion PD. University departments of general practice: a changing scene. Br J Gen Pract 1996; 46: 35-6.

94. Byrne PS. University departments of general practice and the undergraduate teaching of genera practice in the United Kingdom in 1972. J R Coll Gen Pract 1973; 23 (Suppl): 1S$12 \mathrm{~S}$.

95. Oswald N, Jones S, Date J, Hinds D. Long-term community-based attachments: the Cambridge course. Med Educ 1995; 29: 72-6.

96. Fraser EC, McAvoy BR. Teaching medical students at Leicester: the general practice approach. Med Teach 1988; 10: 209-17.

97. Khan NB, Morai-Young Ch A. Formación de pregrado en Medicina de Familia. En: Gómez Gascón T, Ceitlin J, editores. Medicina de Familia: La clave de un nuevo modelo. Madrid : SemFYC / CIMF, 1997; 213-20.

98. Society of Teachers of Familiy Medicine Working Commitee to develop curricular guidelines for a third-year Family Medicine clerkship. Acad Med 1991; 66: 534-9.

99. Otero Puime A. ¿Por qué no existen departamentos de medicina de familia en nuestras universidades? FMC Formación Médica Continuada en Atención Primaria 1997; 4: 256-61.

100. Murray E, Jinks V, Modell M. Community-based medical education: feasibility and cost. Med Educ 1995; 29: 66-71.

101. Murray E, Jolly B, Modell M. Can students learn clinical method in general practice?: A randomised crossover trial based on objective structured clinical examinations. BMJ 1997; 315: 920-3.

102. Hartley S, Berlin A, Tolley N. Can students learn comparable clinical skills in general practice and hospital settings? Students are discriminating consumers of educational experience. BMJ 1998; 316: 1531-2.

103. O'Sullivan M, Martin J, Murray E. Students' perceptions of the relative advantages and disavantages of communitybased and hospital-based teaching: a qualitative study. Med Educ 2000: 34: 648-55.

104. Grayson MS, Newton DA, Klein M, Irons T. Promoting institutional change to encourage primary care: experiences at New York Medical College and East Carolina University School of Medicine. Acad Med 1999; 74 (1 Suppl.): 9S - 15S.

105. Calvo Corbella E, Lorenzo-Cáceres A. de. ¿ Cómo enseñar a los estudiantes de medicina en el Centro de Salud? Tribuna Docente 2000; 1 (4): 3-13.

106. Lassen LC, Larsen JH, Almind G, Backer P. Medical students experience early patient contact in general practice. Scand J Prim Health Care 1989; 7: 53-6.

107. Bonal P, Cots JM, Martín-Zurro A, Calvo E, Casado V, Gil V, et al. Aportaciones de la Medicina de Familia a las Facultades de Medicina de España. Comunicación al XIV Congreso Nacional de la Sociedad Española de Educación Médica. Educación Médica 1999; 2 (3): 148.

108. Saura J, Gil F, Orozco D, Quirce F, Quirós C. Las I Jornadas Nacionales Universidad y Medicina de Familia [editorial]. Aten Primaria 1995; 15 (2): 69-70.

109. SemFYC. Grupo de Pregrado. Sociedad Española de Medicina Familiar y Comunitaria. Manifiesto: Universidad y Medicina de Familia en España. El Médico 5-XI-1999: 16-7.

110. Grupo de Trabajo de Pregrado SemFYC. La medicina de familia como área de conocimiento. Serie Documentos SemFYC, no 10. Barcelona: SemFYC, 1998.

111. Bonal Pitz P. Departamentos de Medicina de Familia en la Universidad Española. JANO 1995; XLIX (1148); 1689-94.

112. Martín Zurro A. Formación en atención primaria de salud. Rev Clin Esp 1992; 191: 325-32.

113. Martín Zurro A. Algunos aspectos esenciales de la medicina de familia. En: Grupo de Trabajo de Pregrado SemFYC. La medicina de familia como área de conocimiento. Series Documentos SemFYC, nº 10. Barcelona: SemFYC, 1998; 9-12.

114. Schwenk Th L. Community-Based Teaching and academic medical centers: A fragile and uneasy alliance. J Fam Pract 1997; 45: 482-92. 